\title{
Moisture detection in walls trough measurement of temperature
}

\author{
by M. Milazzo, N.Ludwig and G.Poldi
}

Institute of Applied General Physics -University of Milano-Via Celoria 1620133 Milano Italy

\begin{abstract}
Evaporation flux from wall surfaces can be estimated quantitatively by measuring the temperature which depends in a sensitive way on the evaporation rate.' When the equilibrium condition is reached the temperature of surfaces where evaporation takes place is in fact directly correlated to the evaporation rate and it depends on several concomitant heat exchange processes. On the other hand starting from non-equilibrium initial condition the "dynamic" measurement of wall surface temperature as a function of time allows the evaluation of water content in the surface layers. In the Archaeometry laboratory of "Istituto di Fisica Generale Applicata" we performed some experiments on several plaster samples among the ones most traditionally used. We employed a climatic room where environmental parameters (temperature, $\mathrm{RH}$, ventilation and irradiation) are controlled and cooling effects due to evaporation are investigated by thermography both in steady and unsteady conditions.
\end{abstract}

\section{Introduction}

Dampness is one of the main problems for preservation of ancient building. The water contained in the wall can be considered as one of the most important causes of surface degradation. Salts dissolved into the water evaporating from the surface, in fact, crystallise in the pores and the volume increase produces microfractures in the building material. This turns into breakage of the superficial layer in chips which can cause a complete shattering of the wall surface. Other possible damages are black stains, loss of adhesion of the paint film from the wet substrate, detachment of the superficial chips, disintegration of the outer layer, biological infestation [1].

A non destructive method for quantitative evaluation of the evaporation phenomenon can be found by measuring with good precision the variations of temperature which depend in a sensitive way both on the evaporation rate, due to the large water vaporisation heat value, and the water content. The temperature of the surface where evaporation is taking place decreases by a few degrees. The cooling effect (which can reach $100-300 \mathrm{~W} / \mathrm{m}^{2}$ ) can be directly correlated to the evaporation rate [2]. It is also possible to obtain from the same measurements an indicative evaluation of the water content in the superficial layer. The heat capacity of the wall is in fact very much affected by the water content [3-4]. Thermography is important in NDT applied to works of art and buildings in surveying the coldest surface areas where evaporation might occur. We note that even by qualitative measurements we can detect possibly endangered areas [5].

In the Archaeometry laboratory of "Istituto di Fisica generale applicata" we performed some experiments on plaster samples among the ones mostly used by tradition. This way we have only a scale model which can approximately simulate the actual surface conditions. However, these "wall surface" samples were examined in environmental conditions which increase the evaporation process. For that we used a climatic room where environment parameters (temperature, relative humidity $\mathrm{RH}$, ventilation and irradiation) can be controlled and evaporation was investigated starting from initial unsteady conditions until steady conditions had been reached. At the same time the environmental quantities which affect primarily the heat exchange terms besides the evaporation (ventilation, direct irradiation) were controlled in order to keep their values as low as possible. The evaporation rate, $\Phi_{\mathrm{ev}}$, can be obtained by solving the proper energy balance equation. We obtained a good agreement with experimental values obtained by weighing the samples. Moreover the measurement of $\Phi_{\mathrm{ev}}$ allows a reliable estimation of the water content. 


\section{Wall energy exchange balance}

The wall surface temperature during evaporation is affected also by heat exchange with the environment. In order to obtain an estimation of the evaporative rate a proper model for the energetic balance on the wall surface must be considered which includes ventilation wind speed, air temperature (corresponding convection term: $h\left(T_{\text {air }}-T\right)$ ), irradiation (ingoing term: $+\alpha \sigma \mathrm{T}^{4}$ env; outgoing term: $\left.-\varepsilon \sigma \mathrm{T}^{4}\right)$, and relative humidity $\mathrm{RH}$, which directly affects the evaporative flux rate $\Phi_{\mathrm{ev}}$. In healthy conditions of moisture content, the energy loss in the evaporative process is negligible if considered within the overall energy balance of the wall surface. Moreover the surface temperature variation due to heat conduction from the inner of the wall can be neglected during the time of thermographic scanning. This is in particular true for ancient walls because of their large thickness and the use of solid bricks. Also the energy associated with mass transfer within the wall (water and salts) it is negligible compared to the energy lost by evaporation. In fact the heat carried by a given quantity of water is approximately two orders of magnitude smaller than the energy required for evaporating the same quantity.

In general the energy balance on a wall surface is given by:

$$
-\varepsilon \sigma T^{4}+\alpha \varepsilon_{a} \sigma T_{a}^{4}+h\left(T_{a}-T\right)+k^{*}\left(T_{\mathrm{int}}-T\right)+\alpha_{v i s} P-\lambda_{e v} \phi_{e v}=0
$$

where:

$\varepsilon=$ sample average emissivity;

$\varepsilon_{\text {env }}=$ environment effective emissivity;

$\sigma=$ Stefan-Boltzmann constant $\left(5,57 \times 10^{-8} \mathrm{~W} / \mathrm{m}^{2} \mathrm{~K}^{4}\right)$;

$\alpha_{\mathrm{ir}}=$ absorption coefficient of the wall surface in the thermal infrared "ir" (8000-14000 nm)

$\alpha_{\text {vis }}=$ absorption coefficient of the wall surface in the visible-near infrared "vis" (380-1100 nm)

$h=$ convection exchange coefficient;

$\mathrm{T}_{\mathrm{a}}=$ air temperature;

$k^{*}=$ conduction coefficient;

$\mathrm{T}_{\text {int }}=$ wall inner temperature;

$P=$ power radiated from sun or lamp $\left[\mathrm{W} / \mathrm{m}^{2}\right]$;

$\lambda=$ vaporisation heat of water $2,4-2,610^{6}(\mathrm{~J} / \mathrm{kg})$;

$\Phi_{\mathrm{ev}}=$ evaporation flux $\left[\mathrm{kg} / \mathrm{m}^{2} \mathrm{~s}\right]$.

When eq 1 applies to a situation of intense evaporation, as in this paper, it causes a heat unbalance, $\Delta Q$, corresponding to temperature decrease by a few degrees. The heat loss due to evaporation can be considered constant, while the variations of temperature of the wall surface modify the efficiencies of radiation and convection processes. In particular, the radiation term (which usually is the most effective cooling process) decreases while the heat absorbed by convection increases. In the assumed condition of intense evaporation the initial loss of energy causes a decrease of temperature per unit time dT/dt inversely proportional to superficial density, $m^{*},\left[\mathrm{~kg} / \mathrm{m}^{2}\right]$ and specific heat $c$. Then we obtain the differential equation:

$$
m c \frac{d T}{d t}=-\varepsilon \sigma T^{4}+\alpha \varepsilon_{a} \sigma T_{a}^{4}+h\left(T_{a}-T\right)+k^{*}\left(T_{\mathrm{int}}-T\right)-\lambda_{e v} \phi_{m e v}
$$

which represents the more general form of equation 1 considering unsteady condition. The solution of eq. (2) gives the relationship between temperature and time.

The integration of eq. (2) can be expressed as follows:

$$
t-t_{0}=m c \int_{T_{0}}^{T} \frac{d x}{-\varepsilon \sigma x^{4}-\left(h+k^{*}\right) x+\left(\alpha \varepsilon_{a} \sigma T_{a}^{4}+h T_{a}+k^{*} T_{\mathrm{int}}-\lambda_{e \nu} \phi_{m e v}\right)}
$$

The calculation of the integral giving the final expression of the solution is reported in the appendix. As it is impossible to give the dependence of temperature on time, the temperature 
data measured by the thermography camera at given time intervals were fitted with the inverted relationship:

$$
t=t_{0}+m c F(T)
$$

where $F(T)$ is the solution of the above integral given in the appendix.

\subsection{Evaporation flux computation}

The evaporation flux is determined from eq. (1) corresponding to the situation when all energetic exchange terms give rise to the equilibrium condition where the temperature assumes its final equilibrium value $T_{\alpha}$. So we have:

$$
\phi_{e v}=\frac{-\varepsilon \sigma T_{\alpha}{ }^{4}+\alpha \varepsilon_{u} \sigma T_{a}^{4}+h\left(T_{a}-T_{\alpha}\right)+k^{*}\left(T_{\mathrm{int}}-T_{\alpha}\right)}{\lambda_{e v}}
$$

Values of $h \varepsilon$ and $\alpha$ can be obtained from literature. In order to determine the numerical value of $\Phi_{\mathrm{ev}}$ the coefficient $h$ is the most critical parameter. In fact the errors on the radiative terms tend to compensate each other and, as already noted, the last term corresponding to the internal heat conduction is negligible. For our experiments on samples in the laboratory we can obtain directly $\Phi_{\mathrm{ev}}$ by checking the sample mass variation during the evaporation. This way from eq (5) values of $h+k^{*}$ were obtained, they show good agreement with literature values [10]. Emissivity was determined by reflectance measurements in $3-5 \mu \mathrm{m}$ band.

\section{Materials and methods}

The tested samples of plaster are composed from different mixtures of sand, lime putty, brick powder, and marble powder. They have the same volumes $\left(10 \mathrm{~cm}^{3}\right)$ and transpirating surfaces. To be prepared for experiments, samples of different composition were dampened and placed in airtight containers for 48 hours where the relative humidity was kept at more than $95 \%$ by using saturated salt solution. We used a climatic chamber (fitotron) that allowed to vary environmental data: $10^{\circ}-45^{\circ} \mathrm{C}$ for air temperature, $20-98 \%$ for relative humidity $(\mathrm{RH})$, $0.1-5 \mathrm{~m} / \mathrm{s}$ for ventilation air speed, and $0-50000$ lux for lighting.

The conditions of our experiments were $25^{\circ} \mathrm{C}, 0 \mathrm{lux}, 0.1 \mathrm{~m} / \mathrm{s}$ air speed (in order to minimise the convection effect) and $\mathrm{RH}$ was kept constant at $50 \%$ or $30 \%$. The samples were insulated on the bottom and lateral sides with a waterproof membrane and placed on a balance (precision: $10^{-4} \mathrm{~g}$ ) inside the fitotron. Weight data were collected on a PC every 2 minutes during two days. From these measurements we verified that $\Phi_{\mathrm{ev}}$ can in fact be considered as constant for several hours. In order to estimate the conditions of heat exchange inside the fitotron we added some dry samples of the same size and emissivity. Changes of air temperature cause significant variations of the convection term and dried samples, having no evaporation, allow for the calibration of the convection term. Using an AVIO TVS 2000 thermovision camera we recorded thermographic scans every 15 seconds during almost 20 minutes. Times of this order were in general sufficient to reach the equilibrium temperature. With direct weight determination we checked the evaporation rates obtained from eq (5).

\section{Results}

Evaporation flux.

The values of $\Phi_{\mathrm{ev}}$ for a few different plasters and for a solid brick core are reported in table 1. The values obtained from eq. 5 in equilibrium temperature show a good agreement with those determined from the weight decrease (same table). 
http://dx.doi.org/10.21611/qirt.1998.013

Table 1. Evaporative flux comparison

\begin{tabular}{|c|c|c|c|}
\hline $\begin{array}{c}\text { Samples } \\
\text { (Composition) } \\
\begin{array}{c}\text { g.c.: lime white, s.t.: white sand, } \\
\text { p.m.: white marble powder, c.p.: brick } \\
\text { crushed. }\end{array}\end{array}$ & $\mathbf{R H}$ & $\begin{array}{c}\Phi \\
{\left[10^{-5} \mathrm{~kg} / \mathrm{m}^{2} \mathrm{~s}\right]} \\
\text { by weighing }\end{array}$ & $\begin{array}{c}\Phi \\
{\left[10^{-5} \mathrm{~kg} / \mathrm{m}^{2} \mathrm{~s}\right]} \\
\text { from eq. (5) }\end{array}$ \\
\hline C (1g.c.+1s.t.+2p.m.) & $30 \%$ & 4.73 & 4.29 \\
\hline F (1g.c+3p.m.) & $30 \%$ & 4.66 & 4.62 \\
\hline G(1g.c.+1s.t.+1p.m.+1c.p.) & $30 \%$ & 5.38 & 4.73 \\
\hline H (1g.c.+3c.p.) & $30 \%$ & 4.64 & 4.60 \\
\hline Core Brick A & $30 \%$ & 8.55 & 8.69 \\
\hline Core Brick B & $50 \%$ & 1.54 & 1.23 \\
\hline
\end{tabular}

Water Content

Also the cooling effect was observed on the same series of plaster samples. Two examples of temperatures during cooling processes are shown in figures 1 and 2 . The initial values before evaporation are about the same as for air. They reach the lowest values at equilibrium when evaporation occurs. The initial condition of non-evaporation was obtained by setting up the air $\mathrm{RH}$ at $95 \%$. Solid lines represent the fit of the experimental data obtained with the function $F(T)$-eq. (4)- that contains the parameter mc (heat capacity). The $m c$ values are derived from the best-fit procedure.

The uncertainty of $F(T)$ depends on the accuracy of several parameters contained in its expression (see appendix). The uncertainty depends strongly on the error of convection and conduction coefficient sum value $h^{*}$ while it depends less on the actual emissivity of the dampened sample. In our experiments values of $35-40\left[\mathrm{~W} / \mathrm{Km}^{2}\right]$ were assumed for $h^{\star}$. The error on $h^{*}$ produces an error of about $10 \%$ in $F(T)$ and consequently the same error can be assumed in the evaluation of $m c$. On the other hand, comparison with mc values obtained by direct calorimetric measurements show the same order of agreement.

\begin{tabular}{|c|c|c|}
\hline $\begin{array}{c}\text { samples } \\
\text { (cf. table 1) }\end{array}$ & $\begin{array}{c}\mathrm{W} \\
\text { (direct } \\
\text { meas.) }\end{array}$ & $\begin{array}{c}\mathrm{W} \\
(\mathrm{F}(\mathrm{T}))\end{array}$ \\
\hline $\mathrm{C}$ & $11 \%$ & $(9 \pm 1) \%$ \\
\hline $\mathrm{F}$ & $13 \%$ & $(15 \pm 2) \%$ \\
\hline $\mathrm{G}$ & $16 \%$ & $(14 \pm 2) \%$ \\
\hline $\mathrm{H}$ & $26 \%$ & $(25 \pm 2) \%$ \\
\hline Brick & $4-5 \%$ & $(5.5 \pm 0.5) \%$ \\
\hline
\end{tabular}

We show in table 2 the water content relative

Table 2. water content comparison values given by direct measurements and by our method with the same error (about 10\%)

$$
W=\frac{m_{w e t}-m_{d r y}}{m_{d r y^{\prime}}}
$$

as that obtained in destructive methods of measurements like drilling (e.g. [6]). The choice of samples of plaster normally employed in frescoes is due to the fact that they represent apparently the most critical case of moisture damage. On the other hand for frescoes destructive tests are not possible even in principle.

\section{Conclusive remarks}

The method that we presented proved to be valid in localisation and quantification of evaporation rates by measuring temperature in equilibrium conditions as above defined.

Concerning the relative content of water, the main problem arises in trying to fix exactly the wall thickness that can be involved in the cooling process which is essentially a superficial phenomenon. However the estimated heat capacity value is related only to a layer of one or two $\mathrm{cm}$ thickness: So we have no connections with water contained in the inner part of the wall. Our method of evaluation of water content depends on the possibility of tracing the temperature as a function of time starting when evaporation effect begins. In addition density and specific heat of the wall material must be known. It is evident that these conditions cannot easily be fulfilled in measurement on the field. 
We stress that to keep the evaporation rate as low as possible is the most important problem for fresco conservation, the exact measurement of water content can be considered as less important. In some "on the field" experiments [7-8] we found that the best environmental conditions for measuring evaporation are achieved at low $\mathrm{RH}(<70 \%)$ and no external heating. The main advantage of thermography applied to conservation of frescoes is that it offers a method for early diagnosis on particularly endangered areas.

\section{REFERENCES}

[1] BARONIO (G.), BINDA (L.) - Durabilità di strutture murarie in mattoni pieni. In "Recuperare", n4, 1983, p. 136-141.

[2] MILAZZO (M.), LUDWIG (N.), VILLANI (S.), SOAVE (C.) and C. CICARDI (C.) Applicability of IR thermography to measurement of leaf transpiration. Proc. of "Advanced Infrared Technology and Applications" in "Atti della fondazione Giorgio Ronchi" Florence Italy, Vol. 1, 1994, p. 171-180.

[3] MALDAGUE (X.) - Nondestructive evaluation of materials by infrared thermography. London, Springer-Verlag, 1993, p. 6,7.

[4] GRINZATO (E.), BISON (G.) and MARINETTI (S.) - Moisture evaluation by dynamic thermography data modelling, proc of Thermosense XVI in SPIE Vol. 22451994.

[5] COLANTONIO (A.) - Thermal performance patterns on solid masonry exterior walls of historic buildings. Proc of Thermosense XIX in SPIE Vol. 3056, 1997, p. 87-95.

[6] LUDWIG (N.), MILAZZO (M.) and POLDI (G.) - Misura di umidità superficiale nelle murature mediante termografia, proc. of National Conference on Non destructive test Padova 1997, Italian Association of NDT, p.163-172.

[7] LUDWIG (N.) and ROSINA (E.) - An Investigation on the Effect of Transpiration on Historical Buildings Using Thermographic Analysis, proc. of Non Destructive Testing In Civil Engineering, Liverpool 1997, British Institute of NDT p. 597-610.

[8] LUDWIG (N.) and ROSINA (E.) - Moisture Detection Through Thermographic Measurements Of Transpiration, proc of Thermosense XIX in SPIE Vol. 3056, 1997.

[9] INCROPERA (F. P), DE WITT (D. P.) - Fundamentals of heat transfer $1973 \mathrm{~J}$. Wiley \&Sons New York.

APPENDIX Solution of integral in eq. (5)

$$
\int_{x_{0}}^{t^{*}} \frac{d x}{-\varepsilon \sigma x^{4}-\left(h+k^{*}\right) x+\left(\alpha \varepsilon_{a} \sigma T_{a}^{4}+h T_{a}+k^{*} T_{\text {int }}-\lambda_{e v} \phi_{m e v}\right)}
$$

The analytical solution $F(T)$ of the above integral is a function of $T_{\alpha}$ and $T_{\beta}$. Where $T_{\alpha}$ and $T_{\beta}$ are solutions in $R$ of the polynomial in the denominator. $T_{\alpha}$ is the equilibrium temperature, while $T_{\beta}$, being negative, has no physical meaning. The explicit expression of $F(T)$ calculated between initial temperature $T_{0}$ and $T$ is:

$$
\begin{aligned}
& F(T)=-\frac{1}{\varepsilon \sigma} f\left[-(1+g) \ln \left|T-T_{\alpha}\right|+g \ln \left|T-T_{\beta}\right|+\right. \\
& \left.+\frac{1}{2} \ln \left|T^{2}+p T+q\right|+\frac{\left(2 T_{\alpha}+T_{\beta}\right)+g\left(T_{\alpha}-T_{\beta}\right)}{\sqrt{4 q-p^{2}}} \operatorname{artg} \frac{2 T+p}{\sqrt{4 q-p^{2}}}\right]
\end{aligned}
$$

where:

$$
p=T_{\alpha}+T_{\beta}
$$


http://dx.doi.org/10.21611/qirt.1998.013

$$
\begin{gathered}
q=\frac{-\alpha \varepsilon_{\alpha} \sigma T_{a}^{4}-h T_{\alpha}-k^{*} T_{\mathrm{int}}+\lambda_{e v} \phi_{m e v}}{\varepsilon \sigma T_{\alpha} T_{\beta}} \\
g=\frac{q+2 T_{\alpha} T_{\beta}+2 T_{\alpha}^{2}+T_{\beta}^{2}}{T_{\beta}^{2}-T_{\alpha}^{2}} \\
f=\frac{T_{\alpha}^{2}-T_{\beta}^{2}}{\left(T_{\alpha}^{2}-T_{\beta}^{2}\right)\left(2 T_{\alpha}^{2} T_{\beta}+T_{\alpha} T_{\beta}^{2}+q T_{\beta}\right)+\left(q+2 T_{\alpha} T_{\beta}+2 T_{\alpha}^{2}+2 T_{\beta}^{2}\right)\left(T_{\alpha} T_{\beta}^{2}-T_{\alpha}^{2} T_{\beta}+q T_{\alpha}-q T_{\beta}\right)}
\end{gathered}
$$

$F(T)$ can not be inverted.

temperature (\%)

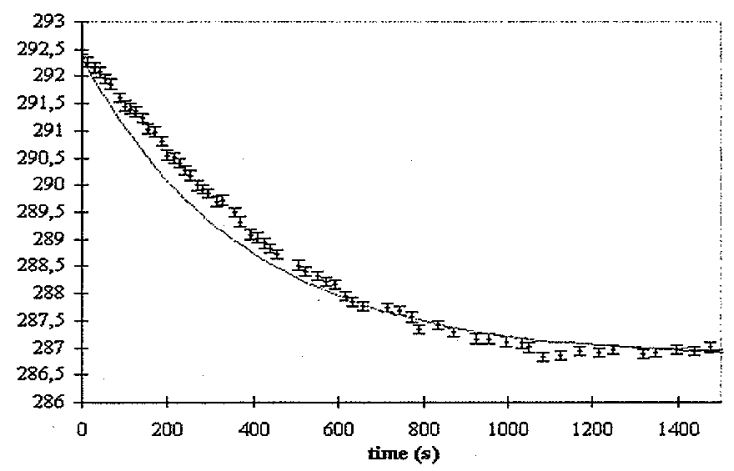

Fig. 1. Cooling of a plaster sample (C.) in $\mathrm{RH}=50 \%$. Solid line obtained with the function $F(T)$ in eq. (4) represents the fit of the experimental data. The best fit of the data assigns the value of $m c$ of the investigated sample.

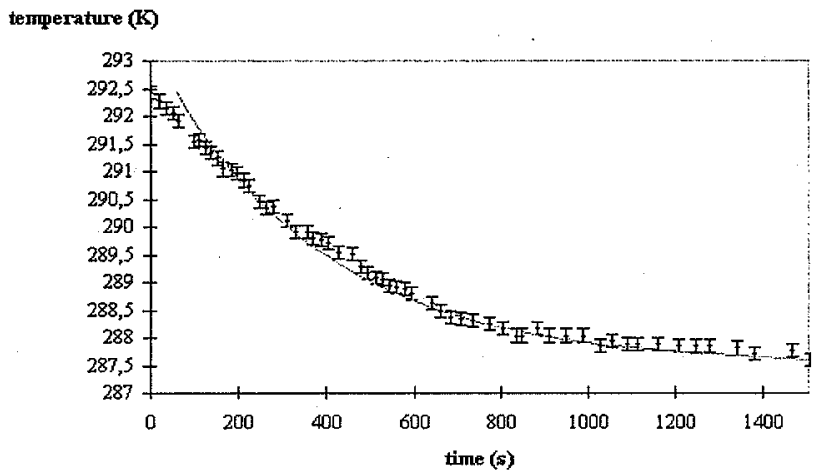

Fig. 2. Cooling of a plaster sample (H.) in $\mathrm{RH}=\mathbf{5 0} \%$. Solid line was obtained with the function $F(T)$ in eq. (4) it represents the fit of the experimental data. The best fit of the data assigns the $m c$ value of the investigated sample. 